\title{
Discorrelated Images
}


This page intentionally left blank 

(C) 2020 Duke University Press

All rights reserved

Printed in the United States of America on acid-free paper $\infty$

Designed by Drew Sisk

Typeset in Portrait Text by Westchester Publishing Services

Library of Congress Cataloging-in-Publication Data

Names: Denson, Shane, [date] author.

Title: Discorrelated images / Shane Denson.

Description: Durham : Duke University Press, 2020. | Includes bibliographical references and index.

Identifiers: LCCN 20I9058965 (print)

LCCN 2019058966 (ebook)

ISBN $978 \mathrm{I} 478009856$ (hardcover)

ISBN 978 I4780IO9I3 (paperback)

ISBN 978I4780I24I2 (ebook)

Subjects: LCSH: Digital images. | Digital cinematography. | Motion pictures-

Production and direction-Technological innovations. | Motion picture industry-

Technological innovations. | Motion picture audiences. | Visual perception.

Classification: LCC TR860.D46 2020 (print) | LCC TR860 (ebook) | DDC 777-dc23

LC record available at https://lccn.loc.gov/2019058965

LC ebook record available at https://lccn.loc.gov/2019058966

Cover art: Karin Denson, Glitchesarelikewildanimals! No. I, 20I5. Acrylic on canvas.

Courtesy of the artist. 
For Amy:

you have taught me

more than anyone else about

how to make sense

of discorrelation 
This page intentionally left blank 